\title{
The length of ribosomal binding site spacer sequence controls the production yield for intracellular and secreted proteins by Bacillus subtilis
}

\author{
Kristina Volkenborn ${ }^{1,4 \dagger}$, Laura Kuschmierz ${ }^{1,2+}$, Nuka Benz ${ }^{1,4}$, Patrick Lenz ${ }^{1}$, Andreas Knapp ${ }^{1,4^{*}}$ (I) \\ and Karl-Erich Jaeger ${ }^{1,3,4}$
}

\begin{abstract}
Background: Bacillus subtilis is widely used for the industrial production of recombinant proteins, mainly due to its high secretion capacity, but higher production yields can be achieved only if bottlenecks are removed. To this end, a crucial process is translation initiation which takes place at the ribosome binding site enclosing the Shine Dalgarno sequence, the start codon of the target gene and a short spacer sequence in between. Here, we have studied the effects of varying spacer sequence lengths in vivo on the production yield of different intra- and extracellular proteins.

Results: The shuttle vector pBSMul1 containing the strong constitutive promoter $\mathrm{P}_{\text {Hpall }}$ and the optimal Shine Dalgarno sequence TAAGGAGG was used as a template to construct a series of vectors with spacer lengths varying from 4 to 12 adenosines. For the intracellular proteins GFPmut3 and $\beta$-glucuronidase, an increase of spacer lengths from 4 to 7-9 nucleotides resulted in a gradual increase of product yields up to 27-fold reaching a plateau for even longer spacers. The production of secreted proteins was tested with cutinase Cut and swollenin EXLX1 which were N-terminally fused to one of the Sec-dependent signal peptides SPPel, SPEpr or SPBsn. Again, longer spacer sequences resulted in up to tenfold increased yields of extracellular proteins. Fusions with signal peptides SPPel or SPBsn revealed the highest production yields with spacers of 7-10nt length. Remarkably, fusions with SPEpr resulted in a twofold lower production yield with 6 or $7 \mathrm{nt}$ spacers reaching a maximum with 10-12nt spacers. This pattern was observed for both secreted proteins fused to SPEpr indicating a dominant role also of the nucleotide sequence encoding the respective signal peptide for translation initiation. This conclusion was corroborated by RT qPCR revealing only slightly different amounts of transcript. Also, the effect of a putative alternative translation initiation site could be ruled out.

Conclusion: Our results confirm the importance of the $5^{\prime}$ end sequence of a target gene for translation initiation. Optimizing production yields thus may require screenings for optimal spacer sequence lengths. In case of secreted proteins, the $5^{\prime}$ sequence encoding the signal peptide for Sec-depended secretion should also be considered.
\end{abstract}

Keywords: Bacillus subtilis, Production optimization, Translation initiation, Ribosome binding site, Spacer

*Correspondence: a.knapp@fz-juelich.de

${ }^{\dagger}$ Kristina Volkenborn and Laura Kuschmierz contributed equally to this work

${ }^{1}$ Institute of Molecular Enzyme Technology, Heinrich Heine University Düsseldorf, Forschungszentrum Jülich $\mathrm{GmbH}, 52425$ Jülich, Germany

Full list of author information is available at the end of the article

\section{Background}

Bacillus subtilis is one of the most important Gram-positive bacteria for the industrial production of recombinant proteins and enzymes [1]. The type strain B. subtilis 168 belongs to the best studied bacteria allowing to devise strategies for production optimization at many different 
stages of transcription, protein biosynthesis and maturation [2]. For example, more than 114 endogenous putative promoters found in $B$. subtilis were assayed for their transcription strengths in different growth phases [3]. Furthermore, libraries of N-terminal Sec secretiondependent signal peptides (SP) can easily be screened to identify the best out of more than 170 different SPs for secretion of a target protein [4-7]. Translation and, in particular, its initiation represents another limiting factor for recombinant protein production [8] which was targeted so far in only few studies with $B$. subtilis.

Translation initiation takes place at the ribosome binding site (RBS) which is located within the $5^{\prime}$ untranslated region (5'UTR) of an mRNA (reviewed for example in $[8$, 9]). The RBS encloses the Shine Dalgarno (SD) sequence, the start codon of the target gene and a short spacer sequence in between. Base pairing of the SD sequence with the anti-SD sequence located at the $3^{\prime}$ terminus of the $16 \mathrm{~S}$ rRNA in the $30 \mathrm{~S}$ small ribosomal subunit initiates the formation of the translation machinery $[10,11]$. Within this initiation complex, the start codon is directed into the ribosomal P-site. Formation of this complex and thus protein production can be improved by so called "strong" SD sequences with high affinity to the respective anti-SD sequence and use of the most frequently occurring start codon AUG [12]. The spacer sequence between $\mathrm{SD}$ sequence and start codon is assumed to bridge the spatial distance between SD sequence and P-site and its optimal length of 7-9nt was determined for the intracellular production of LacZ in B. subtilis [12]. Furthermore, nucleotides flanking the RBS up- and downstream can form mRNA secondary structures that mask the RBS and thereby impede translation initiation [13]. Also, the $5^{\prime}$ sequence of the target gene can influence these secondary structures, and studies of translation initiation in $E$. coli indicate that rare codons in the $5^{\prime}$ sequence of the target gene are not important for deceleration of translation elongation but rather for reducing secondary structures concealing the RBS [14]. Consequently, RBS sequences which were identified as optimal for the production of one target protein cannot be transferred necessarily to another target protein as demonstrated in a study that optimized the production of an intracellular laccase and an extracellular protease [15]. Calculating RNA secondary structures in silico with tools like RNAfold [16] or RBS calculator [17, 18] can help to optimize the production yield of recombinant proteins but they address just one out of several important steps of the complex protein biosynthesis pathway. Here, we present a systematic study to resolve in vivo the effect of the spacer sequence lengths on the production yields of different intra- and extracellular proteins by $B$. subtilis. Furthermore, we have analyzed whether optimal spacer sequence lengths can be predicted and transferred to optimizing the production yield of other proteins.

The study was performed using B. subtilis TEB1030 which is based on the strain 168 derivative DB430 lacking four extracellular (AprE, Bpr, Epr, NprE) and one intracellular protease (IspA) to avoid proteolytic degradation of the target proteins $[19,20]$ and additionally lacking both extracellular lipases LipA and LipB [21]. Genes cloned into the expression plasmid pBSMul1 [22] allowing for high gene copy numbers and strong, constitutive expression [23] under control of promoter $\mathrm{P}_{\text {HpaII }}$. The constructed vector series carries spacers of 4-12nt lengths and we quantified the production of two intracellular and two secreted proteins by SDS-PAGE, activity and split GFP assays. As intracellular proteins, GFPmut3 [24] and the $\beta$-glucuronidase UidA from E. coli (here termed GUS, [25]) and as secreted proteins, Fusarium solani pisi cutinase Cut and B. subtilis swollenin EXLX1 fused to the signal peptides SPPel, SPBsn or SPEpr were produced. Finally, the experimental results from this systematic study were compared to in silico calculated translation initiation rates. The data presented here can serve to optimize protein production using optimal spacer sequence lengths.

\section{Methods}

\section{Media and culturing conditions}

E. coli $\mathrm{DH} 5 \alpha$ [26] and B. subtilis TEB1030 [21] were grown at $37{ }^{\circ} \mathrm{C}$ in shaking flasks with $1 / 10$ volume of $\mathrm{LB}$ medium ( $10 \mathrm{~g} / \mathrm{l}$ tryptone, $10 \mathrm{~g} / \mathrm{l} \mathrm{NaCl}, 5 \mathrm{~g} / \mathrm{l}$ yeast extract) containing either $100 \mu \mathrm{g} / \mathrm{ml}$ ampicillin (E. coli) or $50 \mu \mathrm{g} /$ $\mathrm{ml}$ kanamycin (B. subtilis).

\section{Transformation of E. coli and B. subtilis}

Transformation of chemical competent E. coli $\mathrm{DH} 5 \alpha$ and of B. subtilis TEB1030 protoplasts was carried out as described previously $[27,28]$.

\section{Expression cultures}

For the expression of target genes, a $10 \mathrm{ml}$ overnight culture was inoculated with a single $B$. subtilis transformant and grown at $37^{\circ} \mathrm{C}$ under aerobic conditions. This pre-culture was used to inoculate a $10 \mathrm{ml}$ main-culture to a cell density $\left(\mathrm{OD}_{580 \mathrm{~nm}}\right)$ of 0.05 . The expression cultures were grown at $37{ }^{\circ} \mathrm{C}$ for $6 \mathrm{~h}$ under aerobic conditions. Subsequently, cell density was measured and cells were separated from the supernatant by centrifugation $(21,000 \times g, 10 \mathrm{~min})$ if necessary for further analyses.

\section{Molecular cloning}

Cloning of genes was performed using standard molecular methods [27]. Kits for the purification of nucleic acids were purchased from Analytic Jena (Jena, Germany) and 
enzymes were purchased from Thermo Fisher Scientific (St. Leon-Roth, Germany).

\section{Construction of standard expression plasmids}

Target genes for the construction of the standard expression plasmids (Table 1) were amplified as $\mathrm{NdeI} / \mathrm{XbaI}$ fragments with primers listed in Additional file 1: Table S1 from different templates: GFPmut3 was taken from a modified pEBP41 [29] where we deleted an intrinsic NdeI site by QuikChange $\mathrm{PCR}^{\circledR}[30]$ using the primers $\mathrm{P} 1$ and P2. The GUS gene (uidA) was amplified from E. coli DH5 $\alpha$ genomic DNA. Fusions of EXLX1-11 and cut-11, respectively, with the signal peptide sequences SPepr, SPpel and SPbsn were amplified from a previously constructed signal peptide library [31]. All gene fragments were ligated into the $N d e \mathrm{I} / X b a \mathrm{I}$ hydrolyzed $E$. coliB. subtilis shuttle vector pBSMul1 [22]. This standard expression plasmid contains a 4 nucleotide spacer and is therefore termed pBS4nt in this study.

\section{Construction of expression vectors with different spacer sequence lengths}

The spacer sequence between the SD sequence and the start codon was extended by insertion of adenosines in

\section{Table 1 Plasmids used in this study}

\begin{tabular}{|c|c|c|}
\hline Name & Description & Source \\
\hline pBSMul1 & E. coli-B. subtilis shuttle vector, $\mathrm{P}_{\text {Hpall, }}$ secretion signal (SPlipA), ColE1 repB Km Ampr & {$[22]$} \\
\hline pET22-sfGFP1-10 & $\mathrm{pET} 22 \mathrm{~b}(+)$ containing the truncated SfGFP1-10 gen under control of $\mathrm{P}_{T 7}$ & {$[31]$} \\
\hline pEBP41 & E. coli-B. subtilis-P. putida shuttle vector, $P_{T 7}, P_{x y l}, G m^{\prime}$, GFPmut3 & [29] \\
\hline pEBP41-Ndel & $\begin{array}{l}\text { pEBP41 with deleted Ndel site within the GFPmut3 gene by QuikChange PCR }{ }^{\circledR} \text { using the prim- } \\
\text { ers P1 and P2 }\end{array}$ & This study \\
\hline pBSMul1(SPBox)-SPxxx-cut-11 & $\begin{array}{l}\text { Signal peptide (SP) library based on pBSMul1 containing Hindlll-Xbal inserts of the cutinase } \\
\text { cut of F. solani pisi fused to a GFP11 tag and with different signal peptides (SPepr, SPpel, } \\
\text { SPbsn) }\end{array}$ & {$[31]$} \\
\hline pBSMul1(SPBox)-SPxxx-EXLX1-11 & $\begin{array}{l}\text { Signal peptide (SP) library based on pBSMul1 containing Hindlll-Xbal inserts of the swollenin } \\
\text { EXLX1 of B. subtilis fused to a GFP11 tag and with different signal peptides (SPepr, SPpel, } \\
\text { SPbsn) }\end{array}$ & [31] \\
\hline \multicolumn{3}{|c|}{ Standard expression plasmids (based on pBSMul1 with a 4 nucleotide spacer) } \\
\hline pBS4nt-GFPmut3 & $\begin{array}{l}\text { pBSMul1 containing a } 723 \text { bp Ndel-Xbal GFPmut3 fragment amplified from pEBP41-Ndel with } \\
\text { primers P3/P4 }\end{array}$ & This study \\
\hline pBS4nt-GUS & $\begin{array}{l}\text { pBSMul1 containing a } 1828 \text { bp Ndel-Xbal (uidA) fragment amplified from E. coli DH5a genome } \\
\text { with primers P5/P6 }\end{array}$ & This study \\
\hline pBS4nt-SPepr-cut-11 & $\begin{array}{l}\text { pBSMul1 containing an } 845 \text { bp Ndel-Xbal SPepr-cut-11 fragment amplified from SP library } \\
\text { using primers P7/P10 }\end{array}$ & This study \\
\hline pBS4nt-SPpel-cut-11 & $\begin{array}{l}\text { pBSMul1 containing an } 827 \text { bp Ndel-Xbal SPpel-cut-11 fragment amplified from SP library } \\
\text { using primers P8/P10 }\end{array}$ & This study \\
\hline pBS4nt-SPbsn-cut-11 & $\begin{array}{l}\text { pBSMul1 containing an } 848 \text { bp Ndel-Xbal SPbsn-cut- } 11 \text { fragment amplified from SP library } \\
\text { using primers P9/P10 }\end{array}$ & This study \\
\hline pBS4nt-SPepr-EXLX1-11 & $\begin{array}{l}\text { pBSMul1 containing an } 824 \text { bp Ndel-Xbal SPEXLX1-cut-11 fragment amplified from SP library } \\
\text { using primers P7/P10 }\end{array}$ & This study \\
\hline pBS4nt-SPpel-EXLX1-11 & $\begin{array}{l}\text { pBSMul1 containing an } 806 \text { bp Ndel-Xbal SPpel-EXLX1-11 fragment amplified from SP library } \\
\text { using primers P8/P10 }\end{array}$ & This study \\
\hline pBS4nt-SPbsn-EXLX1-11 & $\begin{array}{l}\text { pBSMul1 containing an } 827 \text { bp Ndel-Xbal SPbsn-EXLX1-11 fragment amplified from SP library } \\
\text { using primers P9/P10 }\end{array}$ & This study \\
\hline \multicolumn{3}{|c|}{ Expression plasmids with extended spacers } \\
\hline pBSxnt-Y & $\begin{array}{l}\text { Spacers ranging in length from } 5 \text { to } 12 \text { nucleotides (xnt) by insertion of adenosines for all } \\
\text { above mentioned plasmids (Y: -GFPmut3,-GUS, -SPepr-cut-11,-SPpel-cut-11,-SPbsn-cut-11, } \\
\text {-SPepr-EXLX1-11,-SPpel-EXLX1-11,-SPbsn-EXLX1-11) }\end{array}$ & This study \\
\hline pBSxnt-SPepr-cut-11_start1 & $\begin{array}{l}\text { pBSxnt-SPepr-Cut-11 with the second putative translational start codon ATG exchanged by } \\
\text { ACG }\end{array}$ & This study \\
\hline pBSxnt-SPepr-cut-11_start2 & pBSxnt-SPepr-cut-11 with the first translational start codon ATG exchanged by ACG & This study \\
\hline \multicolumn{3}{|l|}{ Spacer sequence library } \\
\hline pBS7nt-4N-GFPmut3 & $\begin{array}{l}\text { Spacer library based on pBS7nt-GFPmut3 containing the 7nt long spacer sequence 5'-NNNN- } \\
\text { CAT-3' inserted by QuikChange PCR }{ }^{\circledR} \text { and primers P27/P28 }\end{array}$ & This study \\
\hline pBS7nt-4N-SPpel-cut-11 & $\begin{array}{l}\text { Spacer library based on pBS7nt-SPpel-cut-11 containing the 7nt long spacer sequence } \\
5^{\prime} \text {-NNNNCAT-3' inserted by QuikChange PCR }{ }^{\circledR} \text { and primers P29/P30 }\end{array}$ & This study \\
\hline
\end{tabular}


the spacer sequence of the pBS4nt-SPepr-cut-11 vector using QuikChange PCR $^{\circledR}[30]$ and the primers P11-P26 (Additional file 1: Table S1). The resulting vector series contains spacer sequences with lengths between 5 and $12 \mathrm{nt}$ (as indicated by xnt in the plasmid name). Subsequently, the vector series pBSxnt-SPepr-cut-11 was hydrolyzed using the restriction enzymes $N d e \mathrm{I}$ and $X b a \mathrm{I}$, and NdeI/XbaI fragments of target genes GFPmut3, GUS, SPpel-cut-11, SPbsn-cut-11, SPepr-EXLX1-11, SPpelEXLX1-11 and SPbsn-EXLX1-11 were ligated into these plasmids to construct a vector series with different spacer lengths for each target gene.

\section{Mutagenesis of spacer sequences and spacer library screening}

The spacer sequence AAAACAT of pBS7nt-GFPmut3 and pBS7nt-SPpel-cut-11 was replaced by NNNNCAT $(\mathrm{N}=\mathrm{A}, \mathrm{T}, \mathrm{C}, \mathrm{G})$ preserving CAT and thus the NdeI restriction site of the expression plasmid (see Fig. 1) using QuikChange $\mathrm{PCR}^{\circledR}$ [30] and the primer pairs P27/P28 and P29/P30, respectively (Additional file 1: Table S1). After transformation of E. coli DH5 $\alpha$, about 2000 single clones for each target gene were washed off from agar plates and the plasmid DNA was isolated resulting in the libraries pBS7nt-4N-GFPmut3 and pBS7nt-4N-SPpelcut-11. Libraries were introduced in B. subtilis TEB1030 and 908 clones producing GFPmut3 variants as well as 828 clones producing SPPel-Cut-11 variants were cultivated as pre-culture in microtiter plate scale $(200 \mu \mathrm{l}$ selective medium, $37^{\circ} \mathrm{C}, 900 \mathrm{rpm}, 16 \mathrm{~h}$ ). Main cultures were prepared as 20 -fold dilution of the pre-cultures with fresh selective medium, cultivated for $6 \mathrm{~h}$ under the same conditions and subsequently assayed for intracellular GFP fluorescence or extracellular Cut-11 (lipolytic activity and split GFP assay). The best performing clones were cultivated as biological triplicates in shake flasks (see

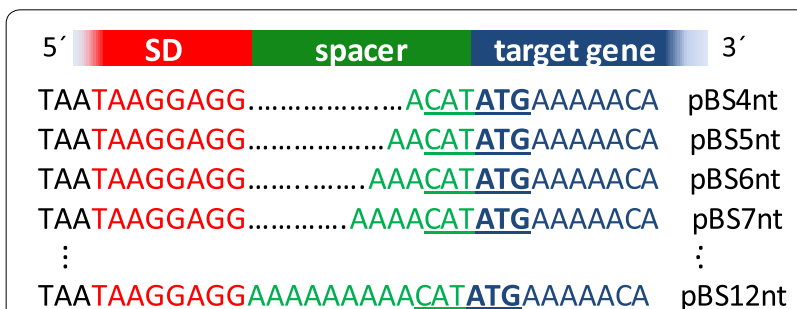

Fig. 1 Nucleotide composition of ribosome binding sites of plasmids pBS4nt to pBS12nt depicting different spacer lengths. Shine Dalgarno (SD) sequence (red), spacer (green) and start of target gene (blue) are highlighted. The cleavage site for Ndel including the start codon (bold) is underlined. Spacer sequences were varied in length from 4 to $12 \mathrm{nt}$ by insertion of adenosines. The original plasmid pBSMul1 is designated here as pBS4nt to indicate the length of the spacer section "Expression cultures" above) with the standard $7 \mathrm{nt}$ constructs as reference.

\section{Construction of plasmids with different translation start sites}

To exchange one of the two different translational start codons (ATG) by ACG in the plasmid series pBSxntSPepr-cut-11, a QuikChange mutagenesis [30] was performed with primer pairs P29/30, P31/32, P33/34, $\mathrm{P} 35 / 36$, P37/38, P39/40, P41/42, P43/44, P45/46 or $\mathrm{P} 47 / 48$ to exchange the first ATG in each of spacer variants (resulting in pBSxnt-SPepr-cut-11_start2), or with primer pair P49/50 to exchange the second ATG in all spacer variants (resulting in pBSxnt-SPepr-cut-11_start1).

\section{Split GFP assay}

The amount of secreted cutinase Cut-11 and swollenin EXLX1-11 was detected by the split GFP assay. The truncated GFP1-10 fragment (detector) was produced by E. coli BL21(DE3) with pET22-sfGFP1-10 as described previously [31]. $20 \mu \mathrm{l}$ culture supernatant was mixed with $180 \mu \mathrm{l}$ detector solution and incubated at room temperature for at least $16 \mathrm{~h}$. Fluorescence was measured using the Tecan Infinite M1000 Pro microplate reader (Tecan, Männedorf, Switzerland). The following parameters were used for fluorescence measurements: $\lambda_{\mathrm{Ex}}=485 \mathrm{~nm}$ (bandwidth $10 \mathrm{~nm}$ ), $\lambda_{\mathrm{Em}}=505-550 \mathrm{~nm}$ (5 nm steps, bandwidth $5 \mathrm{~nm}$, gain 120). The emission maximum at $510 \mathrm{~nm}$ was used for calculation of relative fluorescence units.

\section{Cutinase activity assay}

The lipolytic activity of Cut-11 was measured using the chromogenic substrate $p$-nitrophenyl-palmitate ( $p \mathrm{NPP})$ as described by Winkler and Stuckmann [32]. To prepare the substrate solution, $15 \mathrm{mg} p$ NPP (Sigma-Aldrich/ Merck, Darmstadt, Germany) were dissolved in $5 \mathrm{ml}$ isopropanol and mixed with $45 \mathrm{ml}$ Sørensen buffer $\mathrm{pH}$ 8 (47.22 mM Na $2 \mathrm{HPO}_{4}, 2.77 \mathrm{mM} \mathrm{KH_{2 }} \mathrm{HPO}_{4}, 1.11 \mathrm{mg} /$ $\mathrm{ml}$ gum arabic, $2.3 \mathrm{mg} / \mathrm{ml}$ sodium deoxycholic acid). Culture supernatants were diluted tenfold with $50 \mathrm{mM}$ Tris- $\mathrm{HCl}(\mathrm{pH} 8) .10 \mu \mathrm{l}$ of the diluted culture supernatants were mixed with $190 \mu \mathrm{l}$ substrate solution, incubated at $37^{\circ} \mathrm{C}$ and the change of absorption at $410 \mathrm{~nm}$ was measured for 15 min using the SpectraMax 250 plate reader (Molecular Devices, Biberach an der Riss, Germany). Volumetric activities $(\mathrm{U} / \mathrm{ml})$ were calculated with the molar absorption coefficient of $p \mathrm{NP}\left(15,000 \mathrm{M}^{-1} \mathrm{~cm}^{-1}\right.$ for the used reaction parameters) and subsequently normalized to the cell density $\left(\mathrm{OD}_{580}\right)$. 


\section{GUS activity assay}

Enzymatic activity of GUS was determined with the chromogenic substrate $p$-nitrophenyl-glucuronide ( $p$ NPG, Sigma-Aldrich/Merck, Darmstadt, Germany) as described by Cui et al. [33]. For subsequent cell lysis, $30 \mu \mathrm{l}$ of the GUS expression cultures were mixed with $85 \mu \mathrm{l}$ PBS buffer $(137 \mathrm{mM} \mathrm{NaCl}, 2.7 \mathrm{mM} \mathrm{KCl}, 8 \mathrm{mM}$ $\mathrm{Na}_{2} \mathrm{HPO}_{4} 2 \mathrm{H}_{2} \mathrm{O}, 1.76 \mathrm{mM} \mathrm{KH}_{2} \mathrm{PO}_{4}, \mathrm{pH} 7.4$ ) and $5 \mu \mathrm{l}$ of a $1 \mathrm{mg} / \mathrm{ml}$ lysozyme solution in PBS and incubated at $37^{\circ} \mathrm{C}$ for $30 \mathrm{~min}$. The cell lysate was diluted 20 -fold with PBS buffer and $50 \mu \mathrm{l}$ of the dilution were mixed with $50 \mu \mathrm{l}$ of substrate solution (1.59 mM $p$ NPG in PBS) and incubated at $37{ }^{\circ} \mathrm{C}$ for $2 \mathrm{~min}$. The reaction was stopped by addition of $100 \mu \mathrm{l} 1 \mathrm{M} \mathrm{Na}_{2} \mathrm{CO}_{3}$. The adsorption at $410 \mathrm{~nm}$ was measured using the SpectraMax 250 plate reader (Molecular Devices, Biberach an der Riss, Germany). The volumetric activity $(\mathrm{U} / \mathrm{ml})$ was calculated using the molar absorption coefficient of $p \mathrm{NP}\left(15,301 \mathrm{M}^{-1} \mathrm{~cm}^{-1}\right.$ for the used reaction conditions) and normalized to the cell density $\left(\mathrm{OD}_{580}\right)$.

\section{GFP fluorescence measurement}

The amount of intracellular GFPmut3 was determined by fluorescence measurements. Therefore, $50 \mu \mathrm{l}$ of each expression culture were mixed with $150 \mu \mathrm{l}$ Tris- $\mathrm{HCl}, \mathrm{pH}$ 8. Fluorescence was measured using the Tecan Infinite M1000 Pro microplate reader (Tecan, Männedorf, Swiss). The following parameters for fluorescence measurements were used: $\lambda_{\mathrm{Ex}}=495 \mathrm{~nm}$ (bandwidth $5 \mathrm{~nm}$ ), $\lambda_{\mathrm{Em}}=505-$ $599 \mathrm{~nm}(2 \mathrm{~nm}$ steps, bandwidth $5 \mathrm{~nm}$, gain 100). For the calculation of relative fluorescence units, the emission maximum of GFPmut3 [24] at $511 \mathrm{~nm}$ was used.

\section{SDS-PAGE}

Proteins in cell fractions and supernatants of the different expression cultures were analyzed using SDS-PAGE as described by Laemmli [34]. The extracellular proteins were precipitated using trichloroacetic acid and sodium deoxycholic acid as described in [35]. Briefly, $1 \mathrm{ml}$ of culture supernatant was mixed with $100 \mu \mathrm{l}$ of $1 \%$ sodium deoxycholic acid and incubated on ice for $10 \mathrm{~min} .100 \mu \mathrm{l}$ of cold $40 \%(\mathrm{v} / \mathrm{v})$ trichloroacetic acid solution were added and the samples were incubated on ice for $20 \mathrm{~min}$. Afterwards, the samples were centrifuged at $21,000 \times g$ for $30 \mathrm{~min}$. The supernatant was discarded and the protein pellet was washed with $500 \mu \mathrm{l}$ ice-cold $80 \%$ (v/v) acetone. The protein pellet was dried for $10 \mathrm{~min}$ and subsequently suspended in $50 \mathrm{mM}$ Tris- $\mathrm{HCl} \mathrm{pH} 8$ and $2 \times$ SDS sample buffer $(50 \mathrm{mM}$ Tris- $\mathrm{HCl} \mathrm{pH}$ 6.8, 4\% (w/v) SDS, $10 \%$ (v/v) glycerol, 2\% (v/v) $\beta$-mercaptoethanol, 0.03\% (w/v) bromophenol blue) to an $\mathrm{OD}_{580 \mathrm{~nm}}$ of 15 . Cell fractions were diluted directly in the $2 \times$ SDS sample buffer to achieve an $\mathrm{OD}_{580 \mathrm{~nm}}$ of 15 . All samples were heated to $99{ }^{\circ} \mathrm{C}$ for $10 \mathrm{~min} .15 \mu \mathrm{l}$ of each sample were separated in a $16 \%$ SDS gel in a "Mini Protean II Dual Slap Cell" (BioRad, Munich, Germany) chamber for $15 \mathrm{~min}$ at $100 \mathrm{~V}$ and for $45 \mathrm{~min}$ at $200 \mathrm{~V}$. The separated proteins were detected by staining with Coomassie Brilliant Blue [10\% $(\mathrm{w} / \mathrm{v})$ ammonium sulfate, $1 \%$ phosphoric acid, $0.1 \%(\mathrm{w} / \mathrm{v})$ Coomassie Brilliant Blue R-250, 20\% (v/v) methanol] overnight.

\section{Real-time quantitative PCR for determination of transcript amount}

The influence of spacer lengths on levels of transcript was analyzed for each target gene by RT-qPCR as described previously [36]. RNA was isolated from $1 \mathrm{ml}$ of each expression culture using the NucleoSpin ${ }^{\circledR}$ RNA Kit (Macherey-Nagel, Düren, Germany). Synthesis of cDNA was performed with $1 \mu \mathrm{g}$ RNA with the Maxima First Strand cDNA Synthesis Kit (Thermo Fisher Scientific, St. Leon-Roth, Germany). RT-qPCR was performed using the Maxima SYBR/ROX qPCR Master Mix (Thermo Fisher Scientific, St. Leon-Roth, Germany), 50 ng cDNA of each sample and the real time qPCR primer pairs shown in Additional file 1: Table S1. Gene expression analysis was performed with the REST 2009 software (Qiagen, Hilden, Germany) using the $2^{-\Delta \Delta C T}$ method with an assumed PCR efficiency of 100\% [37, 38]. The expression level of the respective target gene was normalized to the level of the constitutively expressed major sigma factor gene sigA and compared to the expression of the same target gene with a spacer length of $4 \mathrm{nt}$.

\section{In silico analyses}

RNA stability around the translational start site was calculated as minimum free energy (MFE) by the Vienna RNAfold tool $[16,39]$. The Gibbs free energy was calculated for a 39nt window which corresponds to the number of nucleotides covered by the ribosome [40]. This window was shifted between the -50 and the +50 nucleotide position downstream and upstream of the +1 translational start site resulting in 62 individual MFE values for each transcript. The translation initiation rate of each mRNA was calculated using the RBS Calculator v2.0 $[17,18]$. Correlation analysis of translation initiation rates with the experimentally achieved activity or fluorescence data was performed using Microsoft EXCEL 2010 (Microsoft Corporation, Redmond, Washington, USA). For calculation of the Spearman's rank correlation coefficient $\left(r_{s}\right)$ with EXCEL, the rank of each data point was calculated using RANK.AVG function and subsequently the correlation of the ranked data was calculated using the CORREL function. 


\section{Results and discussion}

\section{Construction of vectors harboring spacers of different} lengths

The effect of varying spacer lengths between the Shine Dalgarno (SD) sequence and start codon on the yield of protein produced by B. subtilis was analyzed with a series of vectors based on the expression vector pBSMul1. It contains the strong promoter $\mathrm{P}_{\text {HpaII }}$ [22], a strong SD sequence (TAAGGAGG), and the start codon AUG previously described as being most efficient for protein production [12]. This vector and its derivatives were successfully used in several studies for the production and secretion of recombinant proteins in B. subtilis $[4,5$, 31, 36, 41, 42]. However, pBSMul1 contains a 4nt spacer (ACAT, Fig. 1; for convenience, pBSMul1 is named pBS4nt in this study), whereas a spacer length of 7-9nt is recommended as optimal [12]. In order to identify the optimal spacer length for expression of cytoplasmic and secreted proteins, we stepwise increased the spacer length by QuickChange ${ }^{\circledR}$ PCR in pBSMul1 from 4 to 12 nt by insertion of additional adenosines at the $5^{\prime}$ end of the spacer sequence (Fig. 1).

\section{Optimal spacer length increases product yield of intracellular proteins up to 27-fold depending on the target protein}

To determine the effect of spacer length on the production of intracellular proteins, the genes for the intracellular model proteins GFPmut3 [24], a derivate of the green fluorescent protein from Aequorea victoria, and the $E$. coli $\beta$-glucuronidase UidA [25], here named GUS, were expressed from plasmids with different spacer lengths in B. subtilis TEB1030 [21].
We observed a moderate up to fourfold increase of GFPmut3 fluorescence and an even stronger increase of GUS activity up to 27-fold with increasing length of spacers as compared to the basic constructs with a 4nt spacer (Fig. 2). Accordingly, we also detected increasing amounts of protein by SDS-PAGE (see Additional file 1: Fig. S1A, B) indicating that longer spacers result in the production of an increased amount of protein. The optimal spacer length was at least $7 \mathrm{nt}$ confirming the results of a previous study using LacZ as model protein [12]. However, that study observed a peak for $7 \mathrm{nt}$ spacers with a decreasing productivity for longer spacers whereas our data show that product yields reach a plateau for spacers longer than 9nt. Thus, the optimal length of a spacer apparently depends on the target gene sequence as also indicated by the difference in activity increase factors determined for GFPmut3 (fourfold) and GUS (27-fold).

\section{Optimal spacer length depends on the $\mathrm{N}$-terminal signal peptide for secreted cutinase and swollenin}

Even though the ribosome binds to the 5'UTR upstream of a coding sequence, translation initiation can also be affected by the $5^{\prime}$ end of the coding sequence itself as it is also involved in the formation of RNA secondary structures masking the ribosome binding site [43]. Genes coding for Sec- or Tat-secreted proteins contain a $5^{\prime}$ sequence encoding the $\mathrm{N}$-terminal signal peptide [44]. Remarkably, a given signal peptide can direct the secretion of different recombinant proteins $[45,46]$.

To determine the effect of spacer length on the production of secreted proteins in B. subtilis, we combined three different signal peptides with two secreted model proteins, namely the homologous swollenin EXLX1 and the heterologous cutinase Cut from the fungus F. solani pisi.

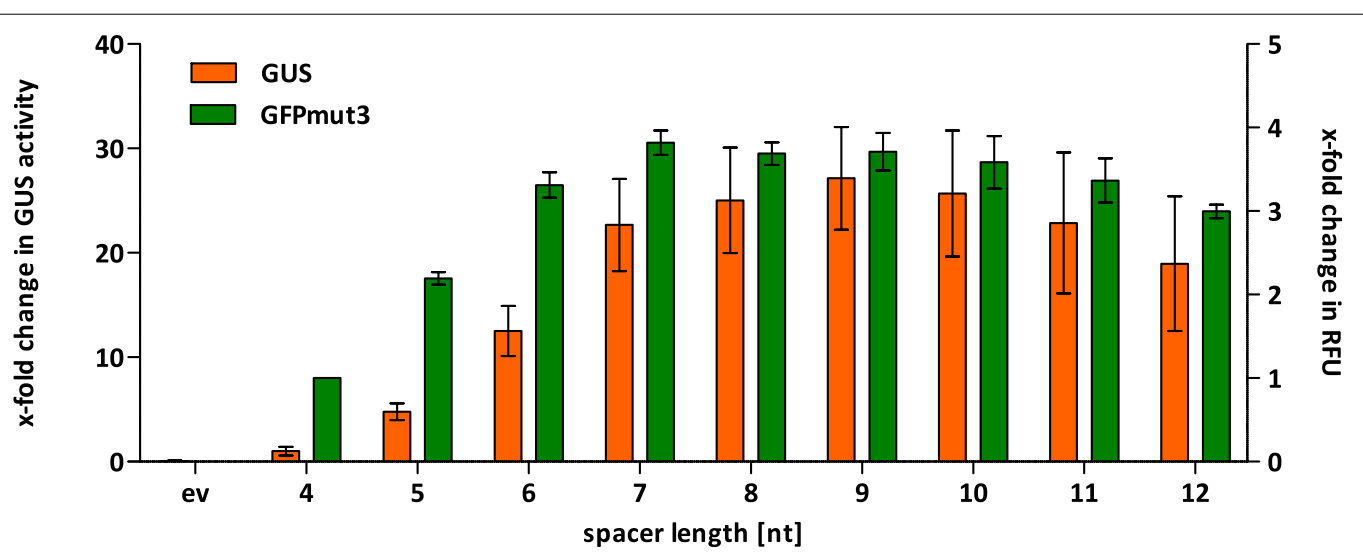

Fig. 2 Determination of optimal spacer length for production of intracellular proteins GUS and GFPmut3. GUS activity and relative fluorescence units (RFU) for GFPmut3 were determined in cell extracts of B. subtilis TEB1030 harboring expression plasmids pBSxnt-GFPmut3, pBSxnt-GUS or the empty vector control pBSMul1 (ev) and calculated as x-fold changes compared to the basic constructs pBS4nt-GFPmut3 or -GUS which was arbitrarily set to 1 
Both proteins were fused in-frame with the B. subtilis signal peptides of the extracellular protease Epr (SPEpr), the pectate lyase Pel (SPPel) and the extracellular ribonuclease Bsn (SPBsn). Signal peptides of Epr and Pel performed well in previous cutinase secretion screenings [4] whereas the signal peptides of Epr and Bsn improved EXLX1 secretion [31]. Both proteins were fused to a C-terminal split GFP tag (GFP11) allowing activity-independent quantification of Cut-11 and EXLX1-11 in vitro [31]. All variants were expressed in B. subtilis TEB1030 from standard plasmid pBS4nt and quantified in the culture supernatant by split GFP assay and additionally lipolytic activity assay for Cut-11 (Fig. 3). Interestingly, the secretion of Cut with the signal peptide from Epr did not result in highest cutinase activity and amount in the supernatant, although it was previously identified as the most suitable signal peptide for the secretion of cutinase [4]. This difference in secretion efficiency might be explained by the different spacer sequence used here (ACAT, see Fig. 1) and in the previous study (ATATT). Nevertheless, all three signal peptides clearly mediated secretion of both model proteins.

All combinations of signal peptides and target genes were expressed with different spacer lengths in $B$. subtilis TEB1030 and proteins Cut-11 and EXLX1-11 were quantified in the culture supernatant by lipolytic activity and by the split GFP assay (Fig. 4). Again, all changes determined by activity and split GFP assays coincided with similar changes in protein amount detected by SDSPAGE (Additional file 1: Fig. S1).

Interestingly, the production of Cut-11 and EXLX111 with the signal peptide SPEpr (Fig. 4a, b) showed a considerable decrease with spacer lengths of 6 and $7 \mathrm{nt}$ followed by a postponed increase of extracellular protein amount with spacer lengths of 10-12nt. This pattern was not described in previous studies dealing with spacer length optimization [12] and differs from the results obtained for both proteins fused to SPPel and SPBsn, where the optimal spacer lengths were 7-9nt with only a slight decrease for spacers with more than 10 nucleotides in length (Fig. 4c-f). Remarkably, the effects of spacer lengths on the production of the target proteins were similar for the same signal peptide indicating that the spacer length has to be adapted predominantly to the $5^{\prime}$ end of the gene of interest which is the SP coding sequence of Sec-secreted proteins.

As suggested in a previous study [42], we also constructed and screened a spacer library for intracellular GFPmut3 and secreted SPPel-Cut-11 with a randomized $7 \mathrm{nt}$ spacer NNNNCAT $(\mathrm{N}=\mathrm{A}, \mathrm{T}, \mathrm{C}, \mathrm{G})$. For this, plasmids pBS7nt-GFPmut3 and pBS7ntSPpel-cut-11 were mutagenized using QuikChange PCR and randomized primers introducing the NNNNCAT spacer instead of the standard AAAACAT spacer. For each protein, over $800 \mathrm{~B}$. subtilis clones were cultivated and assayed in microtiter scale. The spacer sequences of 8 best performing clones were sequenced and the results were re-evaluated under shaking flask conditions with the standard constructs as reference (see Additional file 1: Fig. S2). Interestingly, protein amounts produced by the best performing clones did not exceed those obtained with the standard spacer AAAACAT.

\section{Spacer lengths do not directly correlate with transcript levels}

The observed effects of spacer lengths on the yield of target proteins can have different reasons. On the one
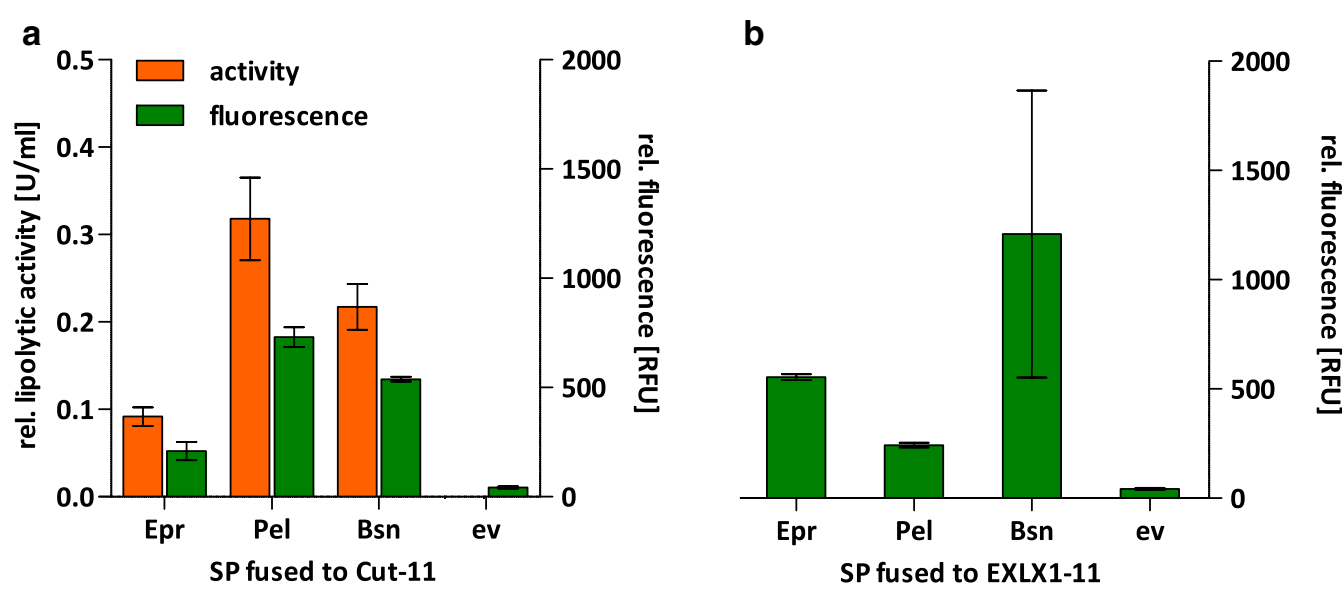

Fig. 3 Extracellular activity and protein amount of cutinase Cut-11 and swollenin EXLX1-11 fused to different signal peptides. Relative lipolytic activity and split GFP fluorescence of Cut-11 (a) and EXLX1-11 (b) produced by B. subtilis TEB1030 harboring expression plasmids pBS4nt-SPepr-Cut-11/-EXLX1-11 (Epr), pBS4nt-SPpel-cut-11/-EXLX1-11 (Pel), pBS4nt-SPbsn-cut-11/-EXLX1-11 (Bsn), or the empty vector control pBSMul 1 (ev) 


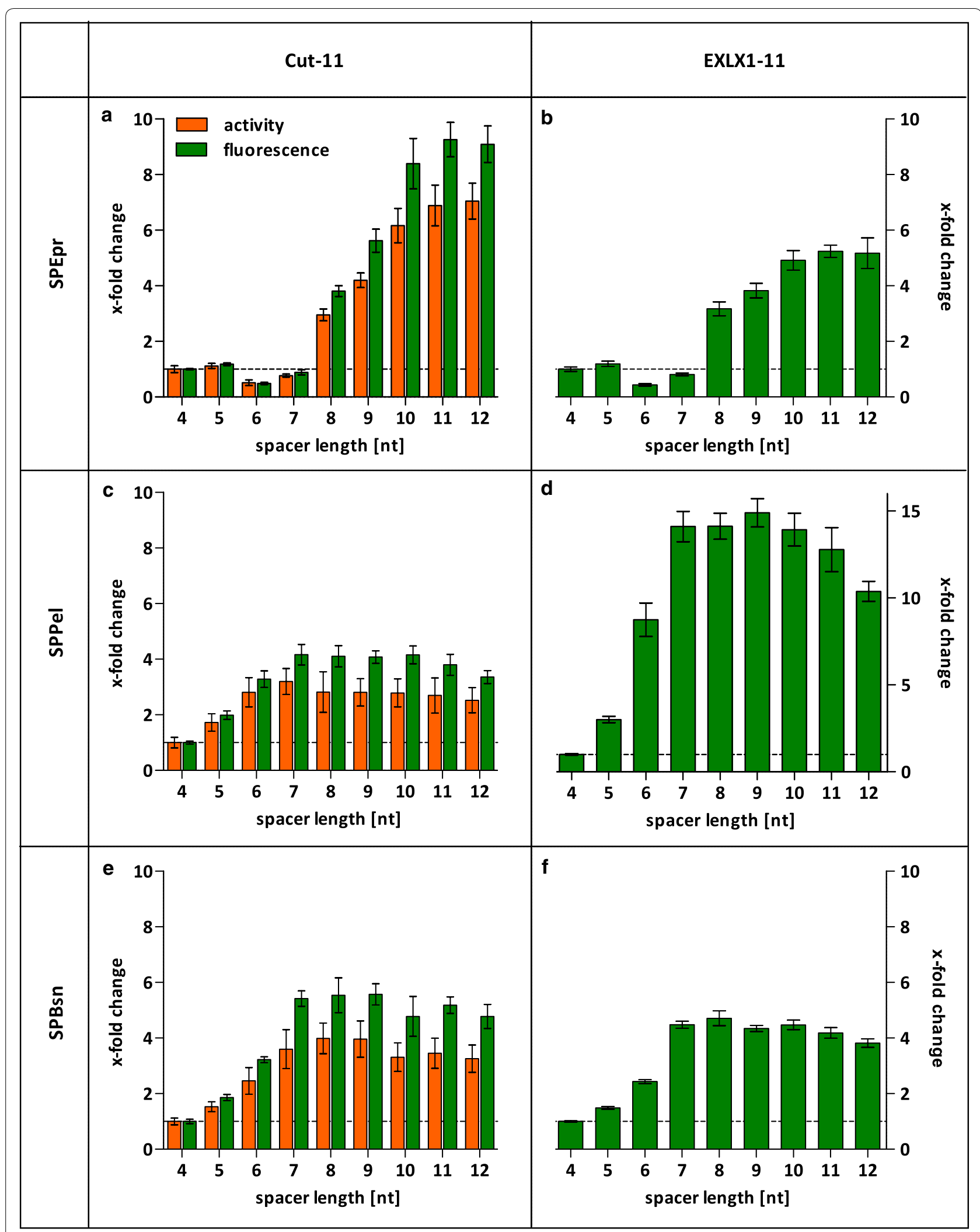

Fig. 4 Influence of spacer length on the production of secreted proteins. X-fold change in Cut-11 activity and amount of Cut-11 and EXLX1-11 proteins determined by split GFP assay in culture supernatants of B. subtilis TEB1030 harboring expression plasmids pBSXnt-SPepr-CUt-11/-EXLX1-11 (a/b), pBSxnt-SPpel-cut-11/-EXLX1-11 (c/d) and pBSxnt-SPbsn-cut-11/-EXLX1-11 (e/f) compared to the respective pBS4nt expression plasmid 
hand, an extension of the spacer length could influence the amount of transcript of the respective gene; on the other hand, the translation initiation rate may be affected. To distinguish between these effects, we have determined the transcript levels for different spacer lengths comprising the basic construct (4nt) and constructs with spacer lengths yielding in decreased (SPEpr 6nt) or increased (GFPmut3 7nt, GUS 10nt, SPEpr 11nt, SPPel $7 \mathrm{nt}$ and SPBsn 8nt) product yields (see Additional file 1: Table S2). Although seven constructs exhibited a significant $(\mathrm{p}<0.05)$ change in transcript amount, the changes were only marginal (max. 3.2-fold) and do not correlate with the observed changes in product yield (see Fig. 4). For example, the transcript amount of 6 nt SPeprEXLX1-11 was significantly increased although product yield was lower than with the basic construct. Thus, it appears that the observed changes in protein production caused by variations in spacer lengths cannot solely be explained by changed amounts of transcript. Consequently, we considered altered translation initiation efficiency as a reason for limited protein production.

\section{Prediction of translation initiation in silico is only partly reliable}

We observed that the relation between spacer length and produced protein yielded in a similar pattern for both extracellular proteins Cut-11 and EXLX1-11 fused to the SPEpr signal peptide (Fig. 4a, b). This led us to conclude that an interaction of the spacer sequence with the $5^{\prime}$ region of the target gene, which encodes the signal peptide, may influence the initiation of translation. It is conceivable that mRNA secondary structures could mask the SD sequence thereby preventing ribosome binding [43]. To predict possible secondary structures masking the RBS, the minimum free energy (MFE) of a dynamic sliding $39 \mathrm{bp}$ window around the translation start was calculated using the Vienna RNA Websuite [39]. This 39 bp window corresponds to the region which is occupied by the $30 \mathrm{~S}$ subunit of the ribosome during translation initiation [40]. A high negative MFE value in this area indicates a possible secondary structure inhibiting translation initiation. The MFE values of all targets with different spacer lengths are shown in Additional file 1: Fig. S3. As the $39 \mathrm{bp}$ window only embraces the signal peptide sequences of the secreted proteins $(66 / 84 / 87 \mathrm{nt}$ for SPpel/SPepr/SPbsn), data for Cut-11 and EXLX1-11 were identical and are pictured only once. The mRNAs of GUS and the SPEpr variants showed very stable structures at the translation initiation site. Those target genes were also observed to need the longest spacers for optimal production yields (see Figs. 2 and 4a, b). Although the secondary structures are weakened by longer spacers in general, MFE values alone seems not to be suitable for prediction of product yields. For example, MFE-based ranking for SPpel predicts almost equally stable secondary structures for all spacer (Additional file 1: Fig. S3) whereas experimental data showed an increase of protein amount (Fig. 4). In addition, the atypical production pattern of SPepr-fused proteins is not reflected by the MFE values for SPepr.

A different method for the in silico analysis of translation initiation is the 'RBS calculator' tool $[17,18]$ which applies a more complex thermodynamic model to calculate the molecular interactions between mRNA and the $30 \mathrm{~S}$ ribosomal complex for the prediction of the translation initiation rate (TIR) of a given gene [17]. We have calculated the translation initiation rates for each target with different spacer lengths (Fig. 5) using the RBS calculator V2.0 and the free energy model version 2.1. Due to the fact that the RBS calculator data is based on 35 nucleotides in front and behind the start codon, translation initiation rates for different target genes with the same signal peptide sequence are again identical. The RBS calculator data show the highest translation initiation rates for constructs with a spacer length of 7 or 8 nucleotides for all target genes (Fig. 5). Based on the RBS calculator data, the optimal spacer lengths for the production of GFPmut3, GUS and the constructs with the signal peptides SPPel and SPBsn were predictable in silico and a priori. In those cases, the variants with the predicted optimal spacer length of 8 nt were among the best producing variants in our experimental setup (see also Figs. 2 and 4) showing a positive correlation with our experimental data $\left(r_{s}\right.$ between 0.70 and 0.97$)$. In contrast, RBS calculator data did not correlate with the experimentally determined data for genes encoding the signal peptide Epr $\left(\mathrm{r}_{\mathrm{s}}<0.15\right)$.

\section{Analysis of a putative alternative translation start site in SPepr}

The atypical production patterns observed for Cut-11 and EXLX1-11 fused to the signal peptide SPEpr could neither be explained by changes in transcript level (see Additional file 1: Table S2) nor by exceptional secondary structures influencing translation initiation (Fig. 5 and Additional file 1: Fig. S3). Inspection of the coding sequence of SPepr identified a putative translation start site 9nt downstream of the annotated start codon (Fig. 6a). The resulting gene product would be a Cut-11 variant shortened by the first three residues of SPEpr. To analyze the effect of this alternative translation start site on SPEpr-Cut-11 production, we replaced the first and the second ATG codon, respectively, of the pBSxnt-SPepr-cut-11 series by ACG (Fig. 6a) which is not accepted as a start codon in B. subtilis [47]. The resulting plasmids pBSxnt-SPepr-cut-11_start1 and 

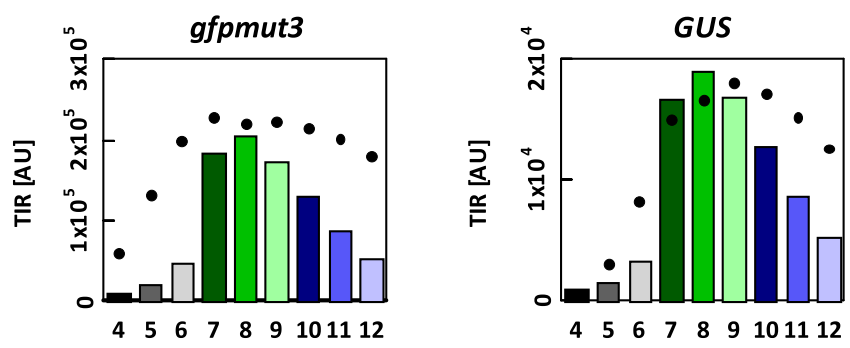

predicted translation initiation rates

(TIR) de pending on spacer length

- production of GFPmut3 or GUS

- production of Cut-11

$\Delta$ production of EXLX-1-11

(da ta derived from Fig. 2 and 4)
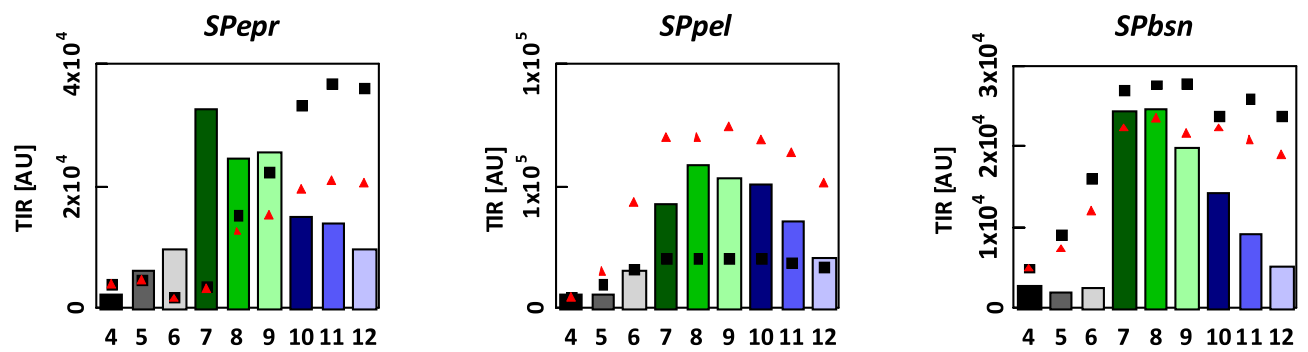

Fig. $\mathbf{5}$ In silico analysis of translation initiation rates. Translation initiation rates (TIR, arbitrary units) for constructs with different spacer lengths were calculated using the RBS Calculator v2.0 [17, 18]. Mean values of experimentally determined changes of GFPmut3, GUS, Cut-11 and EXLX1-11 amounts (shown in Figs. 2 and 4) are indicated
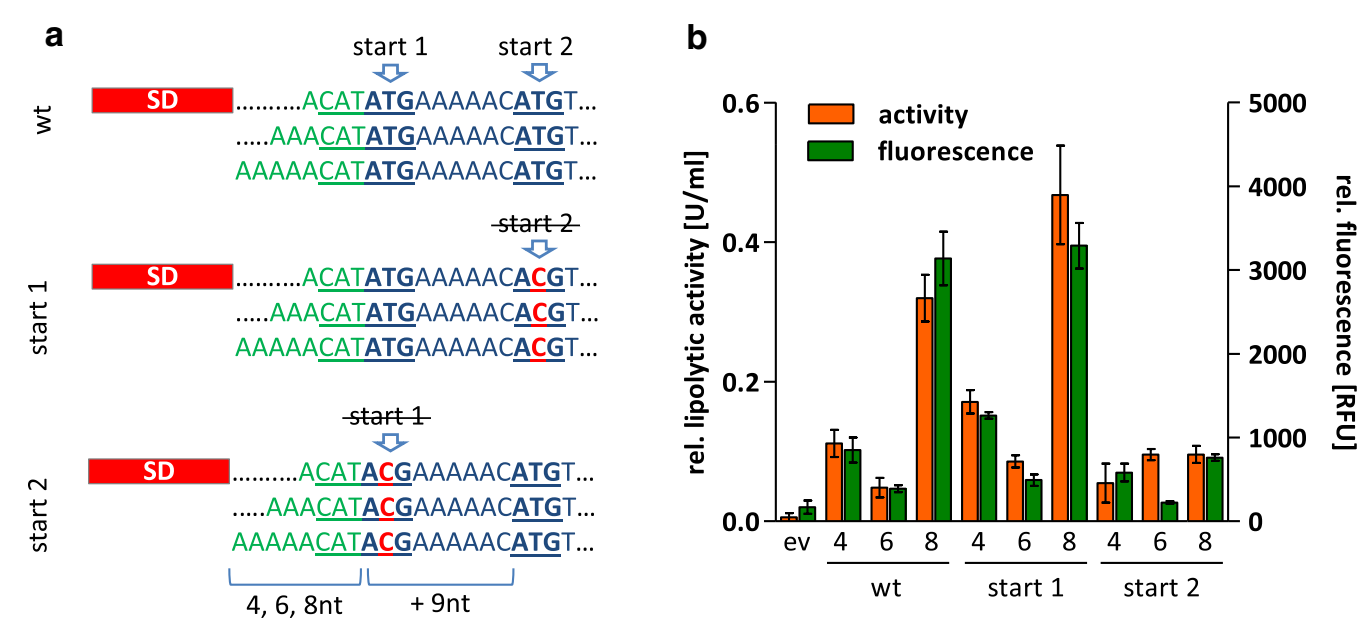

Fig. 6 Effect of an alternative translation start site within the coding sequence of SPEpr-Cut-11. a The original (wt) sequence of SPEpr-Cut-11 contains an ATG codon 9nt downstream of the annotated start codon which could act as alternative translation start site and would result in a Cut-11 variant shortened by the first three amino acids of the SPEpr. Both ATG codons were individually exchanged by ACG to force translation start from the remaining ATG. b Lipolytic activity and amount of extracellular Cut-11 (determined by split GFP assay) which was expressed from the original plasmids (wt) with varying sizes of spacers (4,6 or 8nt) or with first (start 2) or second ATG (start 1) codon exchanged

pBSxnt-SPepr-cut-11_start2 were transferred into $B$. subtilis and extracellular Cut-11 production was quantified in comparison to strains harboring the original plasmids (exemplarily shown for translational start 1 with 4 , 6 and 8nt, and for translational start 2 with putative 13, 15, and 17nt spacers in Fig. 6b). Allowing translation to start only from the putative second start codon (start 2) resulted in low Cut-11 yields independent of the spacer length indicating that this start codon is not responsible for Cut-11 production in the "wild-type" sequence. Interestingly, forcing translation from the first start codon (start 1) still leads to an impaired Cut-11 production with a 6nt spacer but slightly increased the overall Cut11 production by ca. $50 \%$. Thus, the SPEpr-Cut- 11 production pattern cannot be explained by the existence of a second translation start site. However, the second putative start codon together with the preceding purine-rich region (AAAAAC) may probably interact with ribosomes 
thereby impeding translation from the first translational start site as also discussed for additional Shine Dalgarno sequences downstream of the translational start site in $E$. coli [48].

\section{Conclusions}

In this study, we have systematically analyzed the influence of the length of spacers located between the RBS and the start codon on the yields for intracellular (Fig. 2) and secreted proteins (Fig. 4) produced by B. subtilis. Apparently, varying spacer lengths had only limited influence on the transcript amount (see Additional file 1: Table S2) whereas calculated mRNA secondary structures masking the ribosome binding site (Additional file 1: Fig. S3) and translation initiation (Fig. 5) were strongly affected. The spacer sequence together with the $5^{\prime}$-region of the target gene which encodes the signal peptide sequence in case of Sec-secreted proteins [44], constitute the most important part of a target gene with respect to an effective translation initiation as also described recently [13]. Our results further corroborate this observation and pinpoint the importance of signal sequences not only at the level of amino acids [4] but also regarding the respective nucleotide sequences which may directly affect the efficiency of translation initiation.

Interestingly, we observed that protein yields reached a plateau when using spacers longer than at least required for optimal production, whereas literature [12] as well as in silico predictions (Fig. 5) clearly suggest a peak for 7-9nt spacers. A possible explanation is that more efficiently translated proteins could be prone to misfolding and subsequent degradation, e.g. for secreted proteins by proteases HtrA and $\mathrm{HtrB}$ of the general secretion stress system CssRS [49], bringing down a putative production peak for optimal spacers to the mentioned plateau level.

In summary, we have demonstrated that the length of spacer region between SD sequence and transcriptional start side plays an important role if optimal production levels of both intracellular and secreted proteins are envisaged in B. subtilis. In addition, the tested signal peptides seem to not only affect secretion efficiency at the protein level but also the translation initiation at mRNA level.

\section{Supplementary information}

Supplementary information accompanies this paper at https://doi. org/10.1186/s12934-020-01404-2.

Additional file 1: Fig. S1. Influence of spacer length on the production of target proteins. Fig. S2. Influence of spacer composition on the production of GFPmut3 and SPPel-Cut-11. Fig. S3. In silico analysis of mRNA secondary structures. Table S1. Primers used in this study. Table S2. Changes in transcript amounts of target genes with different spacer lengths.

\section{Acknowledgements}

The authors thank Dr. Klaus Liebeton (BRAIN AG, Zwingenberg, Germany) for suggestions and discussions regarding the expression profile of the SPEpr fusion proteins.

\section{Authors' contributions}

KV and LK contributed equally: Both planed and performed most of the experiments and wrote the manuscript. NB and PL planed and performed the analysis of a putative alternative translation start site in SPepr. AK and KEJ planed, coordinated and supervised all experiments and wrote the manuscript. All authors commented on the manuscript. All authors read and approved the final manuscript.

\section{Funding}

The scientific activities of the Bioeconomy Science Center were financially supported by the Ministry of Culture and Science of North Rhine Westphalia within the framework of the NRW Strategieprojekt BioSC (No. 313/323-400-00213). The work of Patrick Lenz was funded by the European Regional Development Fund (ERDF).

\section{Availability of data and materials}

All data generated or analyzed during this study are included in this article and its Additional file 1.

\section{Ethics approval and consent to participate}

Not applicable.

\section{Consent for publication}

Not applicable.

\section{Competing interests}

The authors declare that they have no competing interests.

\section{Author details}

${ }^{1}$ Institute of Molecular Enzyme Technology, Heinrich Heine University Düsseldorf, Forschungszentrum Jülich GmbH, 52425 Jülich, Germany. ${ }^{2}$ Present Address: Environmental Microbiology and Biotechnology-Molecular Enzyme Technology and Biochemistry, University Duisburg-Essen, 45141 Essen, Germany. ${ }^{3}$ Institute of Bio- and Geosciences IBG-1: Biotechnology, Forschungszentrum Jülich $\mathrm{GmbH}, 52425$ Jülich, Germany. ${ }^{4}$ Bioeconomy Science Center (BioSC), C/o Forschungszentrum Jülich, 52425 Jülich, Germany.

Received: 16 March 2020 Accepted: 13 July 2020

Published online: 29 July 2020

\section{References}

1. van Dijl JM, Hecker M. Bacillus subtilis: from soil bacterium to super-secreting cell factory. Microb Cell Fact. 2013;12:3.

2. Nijland R, Kuipers OP. Optimization of protein secretion by Bacillus subtilis. Recent Pat Biotechnol. 2008;2:79-87.

3. Yang S, Du G, Chen J, Kang Z. Characterization and application of endogenous phase-dependent promoters in Bacillus subtilis. Appl Microbiol Biotechnol. 2017;101:4151-61.

4. Brockmeier U, Caspers M, Freudl R, Jockwer A, Noll T, Eggert T. Systematic screening of all signal peptides from Bacillus subtilis: a powerful strategy in optimizing heterologous protein secretion in Gram-positive bacteria. J Mol Biol. 2006;362:393-402.

5. Degering C, Eggert T, Puls M, Bongaerts J, Evers S, Maurer KH, Jaeger K-E. Optimization of protease secretion in Bacillus subtilis and Bacillus licheniformis by screening of homologous and heterologous signal peptides. Appl Environ Microbiol. 2010;76:6370-6.

6. Hemmerich J, Rohe P, Kleine B, Jurischka S, Wiechert W, Freudl R, Oldiges M. Use of a Sec signal peptide library from Bacillus subtilis for the optimization of cutinase secretion in Corynebacterium glutamicum. Microb Cell Fact. 2016;15:208

7. Freudl R. Signal peptides for recombinant protein secretion in bacterial expression systems. Microb Cell Fact. 2018;17:52.

8. Hershey JW, Sonenberg N, Mathews MB. Principles of translational control: an overview. Cold Spring Harb Perspect Biol. 2012;4:a011528. 
9. Unoson C, Wagner EG. Dealing with stable structures at ribosome binding sites: bacterial translation and ribosome standby. RNA Biol. 2007:4:113-7.

10. Wei Y, Silke JR, Xia X. Elucidating the $16 \mathrm{~S}$ rRNA $3^{\prime}$ boundaries and defining optimal SD/aSD pairing in Escherichia coli and Bacillus subtilis using RNASeq data. Sci Rep. 2017;7:17639.

11. Shine J, Dalgarno L. The 3'-terminal sequence of Escherichia coli $16 \mathrm{~S}$ ribosomal RNA: complementarity to nonsense triplets and ribosome binding sites. Proc Natl Acad Sci USA. 1974;71:1342-6.

12. Vellanoweth $\mathrm{RL}$, Rabinowitz JC. The influence of ribosome-binding-site elements on translational efficiency in Bacillus subtilis and Escherichia coli in vivo. Mol Microbiol. 1992;6:1105-14.

13. Sauer $C$, van Ver Loren van Themaat $E$, Boender $L$, Groothuis $D$, Cruz R, Hamoen LW, Harwood C, van Rij T. Exploring the non-conserved sequence space of synthetic expression modules in Bacillus subtilis. ACS Synth Biol. 2018;7:1773-84.

14. Goodman DB, Church GM, Kosuri S. Causes and effects of N-terminal codon bias in bacterial genes. Science. 2013:342:475-9.

15. Hess AK, Saffert $P$, Liebeton $K$, Ignatova Z. Optimization of translation profiles enhances protein expression and solubility. PLOS ONE. 2015; 10:e0127039.

16. Lorenz R, Bernhart SH, Honer Zu Siederdissen C, Tafer H, Flamm C, Stadler PF, Hofacker IL. ViennaRNA Package 2.0. Algorithms Mol Biol. 2011;6:26.

17. Salis HM, Mirsky EA, Voigt CA. Automated design of synthetic ribosome binding sites to control protein expression. Nat Biotechnol. 2009;27:946-50.

18. Espah Borujeni A, Channarasappa AS, Salis HM. Translation rate is controlled by coupled trade-offs between site accessibility, selective RNA unfolding and sliding at upstream standby sites. Nucleic Acids Res. 2014;42:2646-59.

19. Doi RH, He X-S, McCready P, Bakheit N. Bacillus subtilis: a model system for heterologous gene expression. In: Kelly JW, Baldwin TO, editors. Applications of enzyme biotechnology. Boston: Springer US; 1991. p. 261-72.

20. He XS, Shyu YT, Nathoo S, Wong SL, Doi RH. Construction and use of a Bacillus subtilis mutant deficient in multiple protease genes for the expression of eukaryotic genes. Ann NY Acad Sci. 1991;646:69-77.

21. Eggert T, Brockmeier U, Droge MJ, Quax WJ, Jaeger KE. Extracellular lipases from Bacillus subtilis: regulation of gene expression and enzyme activity by amino acid supply and external pH. FEMS Microbiol Lett. 2003;225:319-24.

22. Brockmeier $U$, Wendorff $M$, Eggert T. Versatile expression and secretion vectors for Bacillus subtilis. Curr Microbiol. 2006:52:143-8.

23. Guan C, Cui W, Cheng J, Liu R, Liu Z, Zhou L, Zhou Z. Construction of a highly active secretory expression system via an engineered dual promoter and a highly efficient signal peptide in Bacillus subtilis. N Biotechnol. 2016;33:372-9.

24. Cormack BP, Valdivia RH, Falkow S. FACS-optimized mutants of the green fluorescent protein (GFP). Gene. 1996;173:33-8.

25. Jefferson RA, Burgess SM, Hirsh D. $\beta$-Glucuronidase from Escherichia coli as a gene-fusion marker. Proc Natl Acad Sci USA. 1986;83:8447-51.

26. Woodcock DM, Crowther PJ, Doherty J, Jefferson S, DeCruz E, NoyerWeidner M, Smith SS, Michael MZ, Graham MW. Quantitative evaluation of Escherichia coli host strains for tolerance to cytosine methylation in plasmid and phage recombinants. Nucleic Acids Res. 1989;17:3469-78.

27. Sambrook J, Fritsch EF, Maniatis T. Molecular cloning: a laboratory manual. Cold Spring Harbor: Cold Spring Harbor Laboratory Press; 1989.

28. Chang S, Cohen SN. High frequency transformation of Bacillus subtilis protoplasts by plasmid DNA. Mol Gen Genet. 1979;168:111-5.

29. Troeschel SC, Thies S, Link O, Real Cl, Knops K, Wilhelm S, Rosenau F, Jaeger KE. Novel broad host range shuttle vectors for expression in Escherichia coli, Bacillus subtilis and Pseudomonas putida. J Biotechnol. 2012;161:71-9.

30. Edelheit O, Hanukoglu A, Hanukoglu I. Simple and efficient site-directed mutagenesis using two single-primer reactions in parallel to generate mutants for protein structure-function studies. BMC Biotechnol. 2009;9:61.

31. Knapp A, Ripphahn M, Volkenborn K, Skoczinski P, Jaeger KE. Activityindependent screening of secreted proteins using split GFP. J Biotechnol. 2017:258:110-6.

32. Winkler UK, Stuckmann M. Glycogen, hyaluronate, and some other polysaccharides greatly enhance the formation of exolipase by Serratia marcescens. J Bacteriol. 1979;138:663-70.

33. Cui W, Han L, Cheng J, Liu Z, Zhou L, Guo J, Zhou Z. Engineering an inducible gene expression system for Bacillus subtilis from a strong constitutive promoter and a theophylline-activated synthetic riboswitch. Microb Cell Fact. 2016;15:199.

34. Laemmli UK. Cleavage of structural proteins during the assembly of the head of bacteriophage T4. Nature. 1970;227:680-5.

35. Peterson GL. A simplification of the protein assay method of Lowry et al. which is more generally applicable. Anal Biochem. 1977;83:346-56.

36. Skoczinski P, Volkenborn K, Fulton A, Bhadauriya A, Nutschel C, Gohlke H, Knapp A, Jaeger KE. Contribution of single amino acid and codon substitutions to the production and secretion of a lipase by Bacillus subtilis. Microb Cell Fact. 2017;16:160

37. Pfaffl MW, Horgan GW, Dempfle L. Relative expression software tool (RESTC) for group-wise comparison and statistical analysis of relative expression results in real-time PCR. Nucleic Acids Res. 2002;30:e36.

38. Schmittgen TD, Livak KJ. Analyzing real-time PCR data by the comparative CT method. Nat Protoc. 2008:3:1101.

39. Gruber AR, Lorenz R, Bernhart SH, Neubock R, Hofacker IL. The Vienna RNA websuite. Nucleic Acids Res. 2008;36:W70-4.

40. Beyer D, Skripkin E, Wadzack J, Nierhaus KH. How the ribosome moves along the mRNA during protein synthesis. J Biol Chem. 1994;269:30713-7.

41. Caspers M, Brockmeier U, Degering C, Eggert T, Freudl R. Improvement of Sec-dependent secretion of a heterologous model protein in Bacillus subtilis by saturation mutagenesis of the $\mathrm{N}$-domain of the AmyE signal peptide. Appl Microbiol Biotechnol. 2010;86:1877-85.

42. Liebeton K, Lengefeld J, Eck J. The nucleotide composition of the spacer sequence influences the expression yield of heterologously expressed genes in Bacillus subtilis. J Biotechnol. 2014;191:214-20.

43. Bentele K, Saffert P, Rauscher R, Ignatova Z, Bluthgen N. Efficient translation initiation dictates codon usage at gene start. Mol Syst Biol. 2013;9:675

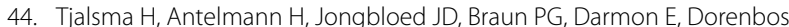
R, Dubois JY, Westers H, Zanen G, Quax WJ, et al. Proteomics of protein secretion by Bacillus subtilis: separating the "secrets" of the secretome. Microbiol Mol Biol Rev. 2004;68:207-33.

45. Mu D, Lu J, Qiao M, Kuipers OP, Zhu J, Li X, Yang P, Zhao Y, Luo S, Wu X, et al. Heterologous signal peptides-directing secretion of Streptomyces mobaraensis transglutaminase by Bacillus subtilis. Appl Microbiol Biotechnol. 2018;102:5533-43.

46. Zhang K, Su L, Duan X, Liu L, Wu J. High-level extracellular protein production in Bacillus subtilis using an optimized dual-promoter expression system. Microb Cell Fact. 2017;16:32.

47. Rocha EP, Danchin A, Viari A. Translation in Bacillus subtilis: roles and trends of initiation and termination, insights from a genome analysis. Nucleic Acids Res. 1999:27:3567-76.

48. Jin H, Zhao Q, Gonzalez de Valdivia El, Ardell DH, Stenstrom M, Isaksson $\mathrm{LA}$. Influences on gene expression in vivo by a Shine-Dalgarno sequence. Mol Microbiol. 2006;60:480-92.

49. Westers H, Westers L, Darmon E, van Dijl JM, Quax WJ, Zanen G. The CssRS two-component regulatory system controls a general secretion stress response in Bacillus subtilis. FEBS J. 2006;273:3816-27.

\section{Publisher's Note}

Springer Nature remains neutral with regard to jurisdictional claims in published maps and institutional affiliations. 